\section{Understanding Covid-19 vaccines}

\author{
By Heather C. Quaile, DNP, WHNP-BC, CSC, SANE, \\ IF, and Tara Thompson, PharmD
}

ealthcare providers have been rolling up their sleeves along with other adults age 65 and older to get their Covid-19 vaccines. Currently, there are three vaccines that have been authorized for use by the US Food and Drug Administration (FDA). This Covid-19 update provides information to help healthcare providers allay some of the fears surrounding vaccination and answer the questions patients are asking.

\section{How Covid-19 attacks and replicates}

Covid-19 is caused by a type of coronavirus. These viruses are named for the crown-like protein spikes on their surfaces. The spike proteins on the outside capsule of the coronavirus lock onto angiotensin-converting enzyme-2 receptors on the surface of human cells via membrane fusion or endocytosis. The virus then enters the cytoplasm of the cell where it fuses with a vesicle and its RNA is released into the cytoplasm. There, the RNA undergoes translation, transcription, and replication to produce more of the virus proteins. These proteins are assembled into the complete mature virion capsule and then released from the cell via exocytosis. ${ }^{1}$

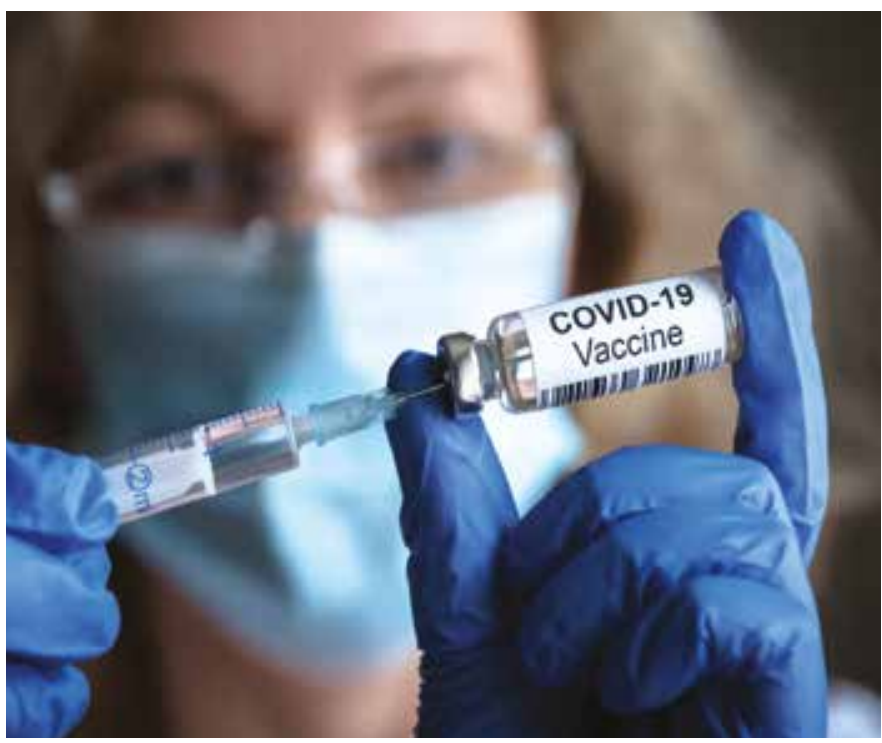

\section{How the body develops an immune response}

The mechanism by which the body develops an immune response to a virus is by detecting the proteins on the virus surface. Once the proteins on the virus surface are detected, the virus capsule is engulfed by specialized antigen-presenting cells, which then show portions of the virus on their surface that activate T-helper cells. These T-helper cells send signals that enable the body's B cells, or fighter cells, to recognize and destroy the viral cells. B cells are activated that make antibodies against the virus and block it from infecting other cells. They also mark the virus for destruction. Cytotoxic T cells then identify and destroy virus-infected cells. These $B$ and $T$ cells now have specific memory to the virus antigen and will patrol the body to protect it if exposed again to the same virus, thus providing at least some degree of future immunity. ${ }^{1}$

\section{How vaccines cause an immune response}

The strategy of every type of vaccine is to expose the body to an antigen that will provoke an immune response but not cause the disease itself. Consequently, the adaptive immune system will recognize the external "villain" and be able to block or kill the virus if exposed to it. Different types of vaccines continue to be tested to determine if they can effectively allow humans to develop immunity to Covid-19. Thus far, two mRNA vaccines and one virus vector vaccine have been authorized for use.

\section{MRNA vaccines}

Although the coronavirus mRNA vaccine was the first of its kind on the market, the use of mRNA has been studied for many decades. In cancer research, mRNA technology has been used to trigger the immune system to target specific cancer cells like melanoma and is now being studied to combat the allergic response. ${ }^{2}$ The mRNA technology is also currently being studied in vaccines designed for influenza, Zika, rabies, and cytomegalovirus. ${ }^{3}$

A major advantage of mRNA vaccines is that they can be formulated synthetically in a lab from readily available materials, which allows for fast vaccine development compared to other types of vaccines. This is important during a swift-moving pandemic. Once the full infectioncausing mechanism of Covid-19 was deduced, researchers knew mRNA technology could be utilized to provide the instructions the cell needed to build the unique spike protein necessary to combat the virus safely and quickly. Within weeks of learning which genes made up the spike protein of the virus, scientists at two major laboratories 
were able to make enough vaccine to test in animals and then in humans. At unprecedented speed, but still developed and tested under the rigorous standards for safety, efficacy, and manufacturing quality set forth by the FDA and just 11 months after the pandemic started, an effective and safely tolerated Covid- 19 vaccine received emergency use authorization by the FDA and was on the market. By the FDA's definition, an emergency use authorization "is a mechanism to facilitate the availability and use of medical countermeasures, including vaccines, during public health emergencies." ${ }^{4}$

The mRNA Covid-19 vaccines have paved the way for widespread immunization. Previously, vaccines have taken at least 4 years to develop. ${ }^{2}$

The technology for developing mRNA vaccines uses only the mRNA genetic material for the coronavirus protein, not the virus itself. The mRNA codes for a diseasespecific antigen, in this case Covid-19, and is used to build those proteins. The mRNA vaccines target the process that cells use to make proteins and trigger an immune response to Covid-19. This newer vaccine concept contrasts with other vaccines that use weakened or inactivated versions or components of the disease-causing pathogen to stimulate the body's immune response to create antibodies. mRNA vaccines stimulate the immune system to make both antibodies (from B cells) and immune system killer cells ( $T$ cells), a one-two punch for coronavirus. Because mRNA can be coded to specific antigens of viruses, it can offer a flexible approach to quickly developing other vaccines should the virus evolve. ${ }^{5}$

\section{Viral vector vaccines}

A Covid-19 nonreplicating viral vector vaccine received FDA emergency use authorization in late February 2021 with distribution beginning In March 2021. The use of viral vectors in medicine is not new. Some vaccines recently used for Ebola outbreaks have used viral vector technology. Apart from being used for vaccines, viral vectors have been studied for gene therapy and to treat cancer. ${ }^{6}$

In this type of vaccine, a small piece of genetic material for the Covid-19 spike protein, not the virus itself, is inserted into a weakened adenovirus. The weakened adenovirus (viral vector) serves as the delivery system. As with mRNA vaccines, the body recognizes the coronavirus protein and elicits an immune response. Antibodies to Covid-19 are produced. The viral vector cannot replicate within cells and is harmless and the spike protein cannot cause Covid-19 without the rest of the coronavirus. ${ }^{6}$ This nonreplicating vector vaccine has two advantages to the currently available mRNA vaccines.
Box. Key points for patient education

- Widespread vaccination is the major means to stopping the Covid-19 pandemic.

- Covid-19 vaccines are highly effective at preventing infection, especially severe infection that can result in hospitalization and death.

- Covid-19 vaccines cannot cause infection.

- Covid-19 vaccines cannot alter a person's DNA (genetic material)

- The CDC recommends receiving Covid-19 vaccine with whichever type is readily available.

- Covid-19 vaccines may cause mild-to-moderate side effects that resolve within a few days.

- Even if fully vaccinated, individuals must continue to practice mask wearing and social distancing for the near future.

The nonreplicating vector vaccine can be stored for at least 3 months in a regular refrigerator unlike the mRNA vaccines that must be shipped and stored at freezing or subzero temperatures. Only one dose is required instead of the two doses needed for the mRNA vaccines. As a third vaccine on the market, it has allowed for increased production and expanded distribution to reach the goal of widespread vaccination.

\section{Discussing vaccine efficacy}

Vaccine efficacy refers to how effective a vaccine is at protecting a person against a specific infection. The two mRNA vaccines have efficacies of $94 \%$ to $95 \%$ after two doses. ${ }^{7,8}$ The virus vector vaccine is $72 \%$ effective in the United States at preventing all Covid-19 infections and $86 \%$ effective in preventing severe cases of the disease after one dose. ${ }^{9}$

It is important for scientists to continue to monitor if emerging variants decrease the efficacy of currently available Covid-19 vaccines. Emerging Covid-19 variants include those believed to have originated in the United Kingdom, Brazil, South Africa, California, and New York. Although data indicate decreased efficacy is possible, experts are optimistic that even if slightly reduced the vaccines will remain significantly effective against the virus.

The Centers for Disease Control and Prevention (CDC) recommends receiving Covid-19 vaccine with whichever type is readily available to the individual. Reported efficacy for each of the three vaccines appears to be the same across all racial and ethnic groups that were included in clinical trials. ${ }^{7-9}$ Healthcare providers should inform patients that although the efficacy rates are impressive, there remains a slight chance of getting 
Covid-19 after receiving any of the vaccines but these cases will likely be much less severe than those in individuals who have not been vaccinated. ${ }^{3}$

\section{Addressing vaccine hesitancy}

Many patients continue to have questions and concerns about the Covid-19 vaccines. Healthcare providers can play a critical role in answering questions and helping patients understand the importance of Covid-19 vaccination (Box). It is anticipated that vaccine supply will increase substantially in 2021 and the goal is for everyone to be able to easily get a Covid-19 vaccine. Some individuals are eager to receive the vaccine right away, while others are hesitant. To promote widespread vaccination, it is necessary to mitigate fear and misconceptions surrounding the vaccine. One commonly expressed concern is the possibility of contracting the virus from the vaccine. Another concern is that the vaccine will in some way alter or interact with the recipient's own genetic material or DNA. The CDC has addressed these concerns ${ }^{3,6}$ :

- You cannot get Covid-19 from the mRNA or viral vector vaccines, as they do not use the live virus that causes Covid-19; and

- Your DNA (genetic material) is not interacted with or altered in any way, as neither mRNA nor the viral vector enters the nucleus of the cell where DNA is kept.

Reactogenicity refers to the production of common or expected reactions or side effects to a vaccine. These reactions are a result of the normal immune response that is generated by a vaccine to build antibodies against a pathogen. ${ }^{78}$ The currently available Covid-19 vaccines may cause mild-to-moderate side effects. The most common side effects include injection site pain, fatigue, and headache. Other side effects experienced include muscle pain, joint pain, chills, and fever. ${ }^{7-9}$ These side effects typically occur within 1 to 2 days of receiving the vaccine and resolve in a few days.7,8,10,11 Healthcare providers should alert patients to these possible side effects and assure them their occurrence does not mean they have the infection. Over-the-counter analgesics and antipyretics can lessen these discomforts if they occur. The anaphylaxis rate with both types of these vaccines is low. ${ }^{7-11}$

\section{Quarantine guidelines and Covid-19 vaccine}

As of early 2021, the CDC updated their quarantine guidelines regarding fully vaccinated persons exposed to someone with suspected or confirmed Covid-19. In- dividuals who have been vaccinated are not required to quarantine if they meet all following criteria ${ }^{3,12}$ :

- Fully vaccinated (ie, $>2$ weeks after completing 2-dose vaccine series or $>2$ weeks after completing single-dose vaccine series);

- Within 3 months of receiving last dose of the vaccination series; and

- Remain asymptomatic following Covid-19 exposure.

If all three criteria are not met, the same 14-day quarantine guidelines established for unvaccinated individuals who have been exposed to anyone with suspected or confirmed Covid-19 should be followed. Even if fully vaccinated and meeting all the criteria, individuals must continue to practice mask wearing and social distancing. Continuing these precautions is prudent to protect others who are not vaccinated because we do not know if a vaccinated individual can harbor the virus in nasal passages and transmit it to others. ${ }^{3}$ These guidelines may bring up the concern as to whether the vaccine only offers 3 months of protection. The 3-month estimate was made out of an abundance of caution. Once there are more long-term data, the estimated length of protection from the vaccine will likely adjust accordingly. 3,12

\section{Conclusion}

Widespread vaccination is the major key to stopping the Covid-19 pandemic. Healthcare providers can provide trusted and accurate information to help patients understand the importance of getting vaccinated and to mitigate vaccine hesitancy based on misconceptions about safety of the vaccine. As well, healthcare providers need to reinforce the continuation of infection prevention by everyone through the use of face masks, social distancing, and handwashing.

\section{References}

1. Callaway E. The race for coronavirus vaccines. Nature. 2020;580:576-577

2. Komaroff A. Why are mRNA vaccines so exciting? Harvard Health Publishing, Harvard Medical School. Harvard Health Blog. December 2020. https://www. health.harvard.edu/blog/why-are-mrna-vaccines-so-exciting-2020121021599.

3. Centers for Disease Control and Prevention. Understanding mRNA COVID-19 vaccines. December 18 , 2020. https://www.cdc.gov/coronavirus/2019-ncov/vaccines/different-vaccines/mrna.html. 
(continued from page 23)

4. U.S. Food and Drugs Administration. Emergency Use Authorization for Vaccines Explained. November 20, 2020. https://www.fda.gov/vaccines-blood-biologics/ vaccines/emergency-use-authorization-vaccines-explained\#: :text=An\%20Emergency\%20Use $\% 20$ Authorization\%20(,COVID\%2D19\%20pandemic.

5. Pfizer. Behind the science: what is an MRNA vaccine? August 25, 2020. https://www.pfizer.co.uk/behind-science-what-mrna-vaccine\#5.

6. Centers for Disease Control and Prevention. Understanding and explaining viral vector COVID-19 vaccines. February 17, 2021. https://www.cdc.gov/vaccines/ covid-19/hcp/viral-vector-vaccine-basics.html.

7. U.S. Food and Drug Administration. FDA briefing document. Pfizer-BioNTech COVID-19 vaccine. Vaccines and Related Biological Products Advisory Committee Meeting. December 10, 2020. https://www.fda.gov/me$\mathrm{dia} / 144245 /$ download

8. U.S. Food and Drug Administration. FDA briefing document. Moderna COVID-19 vaccine. Vaccines and Related Biological Products Advisory Committee Meeting. December 10, 2020. https://www.fda.gov/me$\mathrm{dia} / 144434 /$ download.
9. U.S. Food and Drug Administration. FDA briefing document. Janssen Ad26.COV2.S vaccine for the prevention of COVID-19. Vaccines and Related Biological Products Advisory Committee Meeting. February 26, 2021. https://www.fda.gov/media/146217/download/.

10. U.S. Food and Drug Administration. Emergency Use Authorization for Vaccines to Prevent COVID-19. Guidance for Industry. February 2021. https://www.fda.gov/ regulatory-information/search-fda-guidance-documents/ emergency-use-authorization-vaccines-prevent-covid-19.

11. U.S. Food and Drug Administration. Development and Licensure of Vaccines to Prevent COVID-19. Guidance for Industry. June 2020. https://www.fda.gov/regulatory-information/search-fda-guidance-documents/development-and-licensure-vaccines-prevent-covid-19.

12. Centers for Disease Control and Prevention. Evidence used to update the list of underlying medical conditions that increase a person's risk of severe illness from COVID-19. November 2, 2020. https://www.cdc.gov/ coronavirus/2019-ncov/need-extra-precautions/evidence-table.html.

\section{(continued from page 33)}

countries through Project C.U.R.E. clinics. This would likely require increased understanding of the role of HPV vaccines in disease prevention by clinic partners and local ministries of health. GAVI [Global Vaccine Alliance], in partnership with the Gates Foundation and World Bank, has reduced prices to $\$ 4.50$ per dose, but this price remains a huge obstacle in low- and middle-resource countries. ${ }^{5}$ Fundraising efforts could be aimed at providing a sustainable vaccine supply in these countries and adequate refrigeration facilities when traveling to Project C.U.R.E. clinic sites with unreliable power sources.

Women's health nurse practitioners, nurse midwives, and other specialists may partner with Project C.U.R.E on short-term medical mission trips to fight against disparities. The women of the world deserve no less. Please visit the Project C.U.R.E. website at projectcure.org/ take-action/donate/A to donate supplies or contact our Project C.U.R.E. clinics and college manager Aubrey Buchmann at AubreyBuchmann@projectcure.org.

\footnotetext{
Carolyn Bottone-Post is Assistant Professor at the School of Nursing of University of Northern Colorado in Greeley, Colorado. The author has no actual or potential conflicts of interest in relation to the contents of this article.
}

\section{References}

1. World Health Organization. Cervical cancer. 2021. https://www.who.int/health-topics/cervical-cancer\#tab=tab_1.

2. Centers for Disease Control and Prevention. An estimated $92 \%$ of cancers caused by HPV could be prevented by vaccine. August 22, 2019. https://www.cdc. gov/media/releases/2019/p0822-cancer-preventedvaccine.html.

3. World Health Organization. Human papillomavirus (HPV) and cervical cancer. November 11, 2020. https:// www.who.int/news-room/fact-sheets/detail/humanpapillomavirus-(hpv)-and-cervical-cancer.

4. Brisson M, Kim JJ, Canfell K, et al. Impact of HPV vaccination and cervical screening on cervical cancer elimination: a comparative modelling analysis in 78 low-income and lower-middle-income countries. Lancet 2020;395:575-590.

5. Shinkafi-Bagudu Z. Global partnerships for HPV vaccine must look beyond national income. JCO Glob Oncol. 2020;6:1746-1748.

Web resource

A. projectcure.org/take-action/donate/ 